\title{
Cumulation of the tendency to segregate auditory streams: Resetting by changes in location and loudness
}

\author{
WENDY L. ROGERS and ALBERT S. BREGMAN \\ McGill University, Montreal, Quebec, Canada
}

\begin{abstract}
In four experiments, the accumulation, over time, of a tendency to hear separate high and low streams in a sequence of high $(\mathrm{H})$ and low $(\mathrm{L})$ tones, presented in a galloping rhythm (HLH-HLH-...), was studied. Each trial was composed of two parts, an induction sequence, then a test sequence, with no break between them. The test sequence was always heard at the far left. When the induction sequence and the test sequence were identical, the presence of the induction sequence increased the tendency for the test sequence to split into two streams. However, when the sequences differed in location (cued by differences in interaural timing or intensity over headphones and by loudspeaker placement in a free field) or when they differed in loudness, the accumulation of the segregative tendency was reset, and the test sequence sounded more integrated. When the induction sequence changed in location or loudness in gradual steps toward the value of the test sequence, resetting was much less. It appears that the accumulation of information about streams in different frequency regions is sensitive to sudden changes in parameters, even when they affect the frequency regions equally. This prevents the system from accumulating data across unrelated events.
\end{abstract}

It has been known for some time that the tendency for a listener to segregate a sequence of alternating high and low tones into separate high and low streams increases as the sequence continues (Anstis \& Saida, 1985; Bregman, 1978). This cumulative effect and the stream segregation effect itself have been explained by some authors as being due to the fact that repeated high-low and low-high transitions habituate neural processes that detect frequency transitions (Anstis \& Saida, 1985; van Noorden, 1975). Anstis and Saida also reported that a sudden shift of an alternating sequence of high and low tones from one side of the head to the other led to the temporary loss of separate high and low streams. It appeared that the segregative tendency had been reset and had to build up anew at the new location. Their explanation was that the buildup of segregation was specific to a particular pool of neurons that detected frequency transitions and that those stimulated by the left- and right-ear signals were different. The same resetting of segregation was found when

The names of the authors are in an arbitrary order. These experiments formed part of a PhD thesis submitted by W.L.R. to the McGill University Psychology Department. The General Discussion was written by A.S.B. W.L.R. is presently Director, Clinical Research Group (Adult Division), Douglas Hospital, Verdun, Quebec, Canada. The research was supported by a grant to A.S.B. by the Natural Sciences and Engineering Research Council of Canada and by a McGill Major Fellowship to W.L.R. We thank Pierre A. Ahad for technical assistance and Rhonda Amsel for statistical consulting. Reprints are available from A. S. Bregman, Psychology Department, McGill University, Montreal, PQ, H3A 1BI Canada (e-mail: bregman@hebb.psych. mcgill.ca). the alternating high-low sequence was changed abruptly to a new frequency region; this supported the idea that each region had its own frequency transition detectors.

Rogers and Bregman (1993) used a new paradigm to study the buildup of the strength of the tendency to segregate higher and lower tones into separate streams. They used an alternation of a high $(\mathrm{H})$ and a low $(\mathrm{L})$ tone in a galloping rhythm, which can be schematized as follows: HLH-HLH-HLH- ..., where a dash represents a silence equal in length to a tone $(100 \mathrm{msec})$. Each trial was divided into two parts, a long induction sequence and a short test sequence. The test sequence ( $1.2 \mathrm{sec}$ long) followed the induction sequence (4.8-8 sec long) with no delay. All the test sequences were identical: four iterations of a gallop (HLH-). Listeners judged the degree to which the test sequence segregated. Differences in the segregation of test sequences were examined as a function of the properties of the induction sequences that had preceded them. There is an important reason for using this paradigm: It provides a way to vary the properties of the tones preceding the test sequence in order to tease apart the factors that favor the building up of a tendency to segregate. At the same time, the properties of the test sequence (whose segregation is being judged) are held constant.

Different types of induction sequence were used, and their effectiveness in inducing segregation in the test sequence was evaluated. A number of types of induction sequence were tested by Rogers and Bregman (1993). These included a sequence of high tones only presented at various rates, a single long continuous high tone or white noise burst, a monotic or diotic sequence of HLH- gal- 
lops, gallops with white noise backgrounds, and gallops that were identical to the test sequence in one ear but disguised by tones in the other ear. They made the following observations and conclusions.

1. A high-tones-only induction sequence, containing no frequency transitions, led to an amount of segregation about as large as that induced by a galloping induction sequence. Therefore, there was no support for the theory of Anstis and Saida (1985) or of van Noorden (1975) that stream segregation is caused by the habituation of frequency transition detectors.

2. A temporally irregular high-tones-only induction sequence led to the same amount of segregation as a regular predictable one in which the spacing and rhythm of high tones in the induction and test sequences were identical. This argues against the theory of Jones (1976) that sequential predictability is responsible for the integration of a stream.

3. A right-ear monotic gallop was used for the test sequence. Yet, even if the induction sequence was identical to it and in the right ear (i.e., stimulated the same population of neurons), if the induction sequence also contained, in the contralateral ear, tones whose arrangement caused the subjective loss of the gallop in the induction sequence (i.e., disguised it), there was no induction of streaming to the test sequence. This argued against the claim that continued stimulation of a fixed population of neurons (in the right ear) built up segregation.

4 . When the test sequence was presented only to the right ear, a right-ear induction sequence induced much more segregation than a binaural one did, even though a binaural sequence contains, in the right ear, a galloping sequence. Again, this argued against the idea that the tendency toward stream segregation builds up in ear-specific neural populations.

In the studies of Anstis and Saida (1985), all changes between the ears were sudden, and, in the research of Rogers and Bregman (1993), the transition between induction and test sequences was also sudden. Gestalt psychology assigns a special role to sudden discontinuities in establishing boundaries between perceived objects. The present experiments tested the importance of the suddenness of the change in perceived properties (changes that affect the high and low tones equally) for the tendency to reset the accumulation of information about possible substreams in the sequence. The change between the induction and the test sequences involved (1) spatial location as specified over headphones by either interaural timing cues (ITCs) or interaural intensity cues (IICs), (2) spatial location of loudspeakers, or (3) overall intensity in diotic induction and test sequences.

\section{EXPERIMENT 1}

\section{Method}

Subjects. Ten young adults served as paid subjects. All were university students or staff and reported normal hearing.

Experimental task. On each trial, the subjects listened to an induction sequence, immediately followed by a test sequence (the transition between induction and test sequences was not signaled) Van Noorden (1975) found that attentional factors are important when measuring streaming effects: It is almost always easy to listen for and hear out the individual streams, but the ability to maintain the perception of an up-and-down gallop pattern is highly sensitive to the tone repetition rate and the size of the frequency separation. Therefore, in order to effectively study what makes a coherent sequence split, the subjects were instructed to try to hear a gallop in the test sequence. There was a 6.4-sec intertrial interval.

The subjects were required to judge whether they were able to hear the gallop at the end of the test sequence. Their responses were made on a rating scale, numbered from 1 to 8 , where a 1 indicated with great certainty that a gallop could not be perceived, an 8 indicated with certainty that a gallop could be perceived, and intermediate numbers represented lower degrees of certainty.

The frequency separation between the high and low pure tones was adjusted from trial to trial according to the subject's responses (see details below). The frequencies of the high and low tones in the induction sequence were always the same as those for the high and low ones in the test sequence.

Stimuli. The laterality of the test sequences was held constant at the extreme left of the auditory field, but the lateralities of the induction sequences were varied, and the effects of their lateralizations on the streaming of the test sequences were measured. The induction and test sequences were galloping rhythms of pure tones, as illustrated in Figure 1. Each tone had a 10 -msec onset and offset and an 80 -msec steady state ( $100 \mathrm{msec}$ total). The galloping sequence can be viewed as a regularly alternating high-low sequence in which every other $L$ tone has been replaced by a silence $(-)$. Apart from this replacement, there were no other silences in the sequence. Accordingly, each cycle (HLH-) lasted for $400 \mathrm{msec}$ The induction sequence contained 12 cycles $(4.8 \mathrm{sec})$, and the test sequence contained 3 cycles $(1.2 \mathrm{sec})$.

The extreme left lateralization of the test sequence was produced by an interaural time delay of $0.7 \mathrm{msec}$ in the right ear. This value was the closest approximation available at a $20-\mathrm{kHz}$ sampling rate for the value of $0.69 \mathrm{msec}$, the value given by Moore (1982, p. 151) for a sound directly to one side of a listener. The induction sequence preceding each test sequence contained cycles of the gallop pattern with a high-low frequency separation matching that of the test sequence but differing from it in apparent spatial location.

Experimental conditions. There were six different types of induction sequences, chosen to provide gradual, sudden, and random

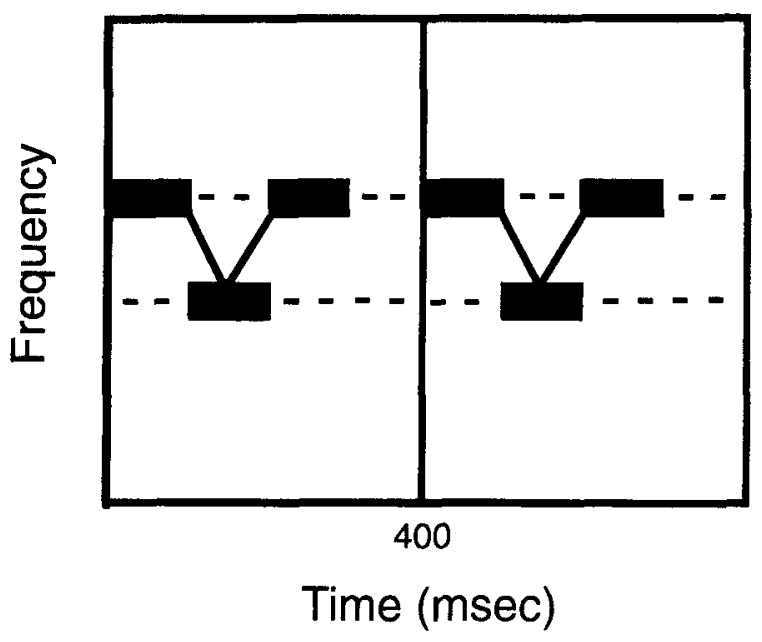

Figure 1. Two cycles of the galloping pattern of tones used in the induction and test sequences. 
changes of lateralization between the induction and the test sequences.

1. No change. The induction sequence was presented at the extreme left - that is, with the same lateralization as the test sequence. This condition was expected to produce the most segregation.

2. Gradual change. The beginning of the induction sequence was lateralized at the extreme right of the auditory field, and the lateralization was changed by about $15^{\circ}$ of azimuth on each cycle, so that the gallops were heard to move gradually from right to left. The time delays (given relative to the time of arrival of sound at the left ear) were $-0.7,-0.55,-0.45,-0.35,-0.25,-0.15,0,0.15$, $0.25,0.35,0.45,0.55$, and $0.7 \mathrm{msec}$. Thus, there were 13 different lateralizations for each frequency separation in the experiment. It was predicted that, when change was gradual, the auditory system would treat the tones of both the induction and the test sequences as belonging to a single sound source that was changing slowly in location; maintenance of a one-source percept should favor cumulative induction of streaming, as in the no change condition.

3. Random change. The apparent spatial location changed randomly to any of the 13 possible values from cycle to cycle of the induction sequence. Each trial of each subject was independently randomized. This condition was expected to induce streaming of the test sequence in an amount intermediate to the no change and the control conditions. On the one hand, the changes were frequent, but sometimes the gallops appeared at the location of the test sequence.

4. Sudden right-to-left. The induction sequence had an apparent location at the extreme right.

5. Sudden center-to-left. The induction sequence had an apparent location in the center of the auditory field (time of arrival the same at both ears). The fourth and fifth conditions were both included because it was not known which of the two induction sequences would be heard as more different, for purposes of auditory organization, from the test sequence. The change from right to lef $t$ makes for a greater absolute displacement, whereas the change from center to left makes for a greater displacement from the center.

6. Control. The induction sequence was replaced by a single long burst of dichotic white noise, containing no gaps, of the same total duration as the induction sequence in Conditions $1-5$. The noise in the two ears was uncorrelated, so that the subject's attention was not focused on any particular location in auditory space. The control condition was expected to have the least induction effect on the test sequence, since the frequencies that would follow during the test sequence were not presented any more frequently than any other frequencies. We could have replaced the induction sequence with a silent interval instead of the noise in the control condition, but we judged that the presence of the noise made the trial more subjectively similar in overall timing to the other conditions. In addition, the results of Bregman (1978) suggested that white noise would not facilitate streaming any more than silence would. (The 1978 experiment actually showed that $88-\mathrm{dB}$ white noise had the same effect as silence in allowing a streaming tendency to decay after the presentation of a short sequence of alternating high and low tones.)

All the subjects were tested under all six levels of induction condition type, with nine trials at each level.

Training. The subjects were first shown a diagram of the gallop pattern, and the experimenter tapped out the rhythm. They then listened as often as they liked to sample stimuli chosen as unambiguous examples of a segregated sequence (high and low tones separated by 19 semitones), described to the subject as a nongallop, a coherent gallop (high and low frequencies 3 semitones apart), and a burst of white noise. Then, they were given two practice trials for each induction condition on the experimental task described above.

To summarize, the amount of segregation induced by the induction sequence was expected to be greatest with the no change condition and the least with the cont rol condition. Both of the sudden changes were expected to be similar in effect to the control condition, and the gradual change similar in effect to the no change condition.

Measurement of induction. When a subject is trying to hold a sequence together, any increase in the frequency separation of the high and low tones causes stream segregation to get larger. That is, the sequence is more likely to segregate into separate high and low streams. This effect was employed to measure the degree of segregation that was induced by the induction sequence. Both the induction sequence and the test sequence always had the same frequencies of high and low tones. The frequency differences within each of these sequences, viewed separately, should gradually build up a tendency for its high and low tones to segregate. In addition, the similarities between the properties of the test and the induction sequences should determine whether the tendency toward segregation, accumulated during the induction sequence, transfers to the test sequence, augmenting the latter's tendency to segregate. The greater the transfer (or induction), the less frequency separation (for the overall induction-test sequence) should be required before the subject would report segregation by the end of the test sequence. Therefore, the frequency separation was adjusted from trial to trial (always keeping the frequency separation the same in the induction and test sequences). The dependent variable was the frequency separation at which the galloping percept was lost (the temporal coherence boundary of van Noorden $(1975,1977)$.

Ten different frequency separations were possible between the high and low tones of the sequences: from 5 to 14 semitones. The high tone was always fixed at $699 \mathrm{~Hz}$; the low ones ranged from 311 to $523 \mathrm{~Hz}$. This provided a range of separations that was expected to include both segregation and coherence by the end of the test sequence for each subject under each condition. On the first trial of each condition, the subject heard sequences with a 9-semitone separation. On the next trial of that condition, the separation was adjusted, depending on the response for the previous trial in that condition. If the response had indicated coherence (gallop perceived-ratings 5-8), the frequency separation of the current trial was made 1 semitone larger, whereas if the response had indicated segregation (gallop not perceived-ratings 1-4), the separation was made 1 semitone smaller. The trials on different conditions were interspersed randomly (randomization in blocks of six, with independent randomizations for each block and each subject); the program remembered the performance on each trial so as to be able to adjust the frequency separation separately for each condition.

Over the course of the experiment, the separations were expected to move toward a value, for each condition, that represented a boundary between segregation and coherence. Whether the separation reached the asymptotic value was not important for determining differences. Since all conditions had the same number of trials and started at the same separation, any difference in the final values would reflect differences in the induction of segregation. However, if a group of conditions all reached values close to the maximum frequency separation ( 14 semitones), it would be possible that this similarity was due to a ceiling effect. For this reason, similarities among sets of means close to 14 semitones were not taken to be evidence that the effects of induction are the same in these conditions.

Apparatus and Stimulus Parameters. The stimuli were digitally synthesized, using the MITSYN signal-processing system (Henke, 1987). The timing and randomization of trials, as well as data collection, were handled by a separate program. The sampling rate for digital synthesis and playback was $20 \mathrm{kHz}$. The level of the tones was set to $65 \mathrm{dBA}$, and that of the white noise to $51 \mathrm{dBA}$. Signals were low-pass filtered at $5 \mathrm{kHz}$ by a Rockland 851 filter, with a roll-off of $48 \mathrm{~dB} /$ octave. They were presented over Sony MDR-V7 stereo headphones, in an Industrial Acoustics Company audiometric testing room, Model 1202 . The subjects received prompts on a video display terminal and entered their answers on a key- 
board, both located inside the chamber. An IBM-compatible computer outside the chamber controlled the experiment. For calibration of intensity, a General Radio Company Model 1565B sound level meter, a flat-plate headphone coupler, and A weighting were used. It was carried out on continuous pure tones at the frequency of the upper tones of the test sequence gallops.

\section{Results}

The dependent variable was the average, for each condition, of the frequency separations used on the last two replications of the trials for that condition. Higher values indicate that it required a larger frequency separation before the subjects abandoned the gallop interpretation of the sequence; this indicated that the induction sequence was less effective in inducing segregation. Conversely, low scores reflect a bias for stream segregation. A oneway (induction condition) repeated measures analysis of variance (ANOVA) was conducted. A preliminary analysis of the data showed homogeneity of covariance so the Greenhouse-Geisser (1959) correction used in Experiments 2-4 was not employed. A significant effect of induction condition was found $[F(5,45)=8.754, p<.0001]$. The means for the no change, gradual, random, sudden right-to-left, sudden center-to-left, and control induction conditions were (respectively) 8.45, 9.6, 10.05, 10.7, 11.5 , and 13.25 semitones. The results are shown in Figure 2, with the dependent variable (referred to as the segregation boundary) plotted against induction condition type.

\section{Effect of lateralization change: Interaural timing cues}

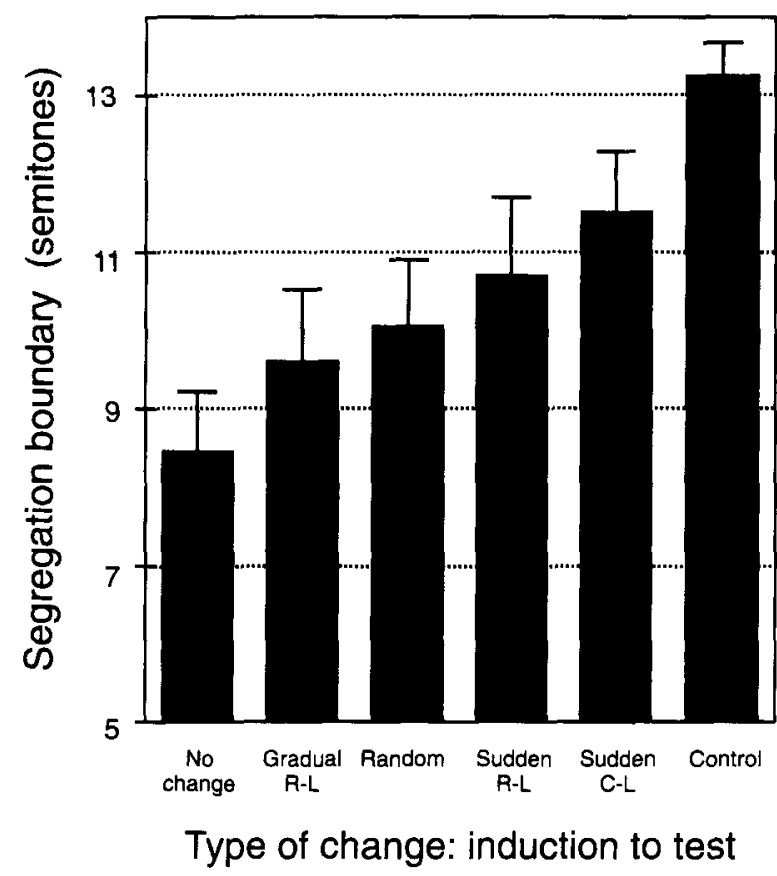

Figure 2. Results of Experiment 1. Segregation boundary following six types of change from induction to test sequence, using interaural timing cues to control lateralization. Higher values indicate a lower induction of segregation. Vertical bars show standard errors.
A planned contrasted of the average of the two sudden change conditions contrasted with the average of the no change and gradual change conditions was significant $[F(1,9)=4.21, p=.0068]$. However, the planned comparison between the two sudden change conditions showed no significant difference $[F(1,9)=0.619, p=.0673]$. A Bonferroni adjusted $t$ test, comparing all pairs of means, was conducted at the .05 level. The control condition was reliably different from all other conditions, except for the two sudden change conditions, but there were no other significant results.

The mean for the control condition was close to the maximum value of 14 , suggesting that there may have been ceiling effects in the experiment. Perhaps, if ceiling effects had been avoided, we might have found a significant difference between the sudden change conditions and the control condition. However, this would simply have shown that, even with sudden changes, some induction of streaming could occur. It would leave unaffected the conclusion that sudden changes induce less streaming than do no change or gradual changes.

\section{Discussion}

Figure 2 shows that, in the no change condition, in which the test sequence was an unmodified continuation of the induction sequence, the subjects segregated the test sequence the most. The white noise control induced the least segregation, with the sudden change conditions and the random change condition next, followed by the gradual change condition. The effect of a sudden change from the center to the left of the auditory field was the same as that for a change from the extreme right to the left, with ITCs. This could be because any sudden change in azimuth over some threshold value is sufficient to cause a resetting of cumulative streaming processes.

We interpret these results as confirming the initial hypotheses. With no change of lateralization or a gradual change, the gallop patterns of the induction and test sequences were evidently treated as part of the same sound source, and the tendency to segregate the high from the low tones was allowed to continue to build up. On the other hand, as the changes from induction to test sequences became more sudden, there was an increasing tendency to treat the test sequence as a new sound source, and the cumulation of the tendency to segregate high from low tones was reset.

Among the conditions employed by Rogers and Bregman (1993) was one (in Experiment 3 ) in which there was a sudden change in localization between induction and test sequences from a centered position (sound in both ears) to a right-side position (sound in the right ear only). They found that this reduced the amount of induction. when compared with a condition in which there was no change. The effect was caused by a sudden change in IICs. The results of the present experiment show a similar effect when the lateralization was produced by ITCs.

This similarity in the effect of two different interaural cues suggests that the effect is related to sudden changes 
in the general property of spatial location of an existing stream. It is important to note, however, that pilot testing of the present experiment failed to find a significant effect of sudden changes in ITCs, using the same method as Rogers and Bregman (1993) - namely, a rating scale with fixed high-low frequency differences. We were forced to go to the present method, in which the frequency separation was adjusted, before we could find clear effects of ITCs. This suggests that a sudden change in ITCs has a smaller effect on resetting the cumulation of frequency information than does a sudden change in IICs.

\section{EXPERIMENT 2}

In this experiment, we again examined how changes in localization between induction and test sequences would reset the cumulation of the tendency to segregate high from low tones in a galloping sequence. This time we used changes in IICs to generate all the types of induction sequence present in Experiment 1.

\section{Method}

The method used in this experiment was identical to that of Experiment 1 , except as noted below.

Subjects. Twenty-three young adults were tested. Data from 3 were not used, due to errors in the experimental procedure.

Stimuli. All the tones were in the range $1867-4192 \mathrm{~Hz}$. These frequencies were chosen to be in a range at which IICs are very effective in specifying location but were kept well below $5000 \mathrm{~Hz}$. This was done because the perception of musical pitch, which may be needed for doing the experimental task, is lost at frequencies above about $5000 \mathrm{~Hz}$ (Attneave \& Olson, 1971). The sampling rate was $16 \mathrm{kHz}$. There were 10 possible frequency separations between the high and the low tones of the gallops, varying from 5 to 14 semitones in 1-semitone steps.

The test sequence was always heard at the extreme left of the auditory field to allow for comparison with Experiment 1. This was produced by an interaural intensity difference of $10 \mathrm{~dB}$, with the left ear receiving the greater intensity. This value was chosen in accordance with values given by Feddersen, Sandel, Teas, and Jeffress (cited in Warren, 1982, p. 35) of actual sound level differences measured at the two ears as a function of azimuth for sinusoidal tones of different frequencies; a difference of about $10 \mathrm{~dB}$ (averaged over frequencies of $1800,2500,3000$, and $4000 \mathrm{~Hz}$ ) is the sound shadow cast by the head for a sound located at $80^{\circ}-90^{\circ}$ of azimuth.

Experimental conditions. The induction sequences differed from the test sequences only in apparent spatial location, cued by interaural intensity difference. They were five of the six types of induction sequence used in Experiment 1 (the random change condition was omitted), except that spatial location was signaled by interaural intensity differences. The five types of sequences were as follows.

1 . No change. Both induction and test sequences were lateralized at the extreme left.

2. Gradual change. The first gallop of the induction sequence was lateralized at the extreme right of the auditory field, due to the $10-\mathrm{dB}$ interaural difference. During the induction sequence, the amplitudes presented to each ear were changed linearly throughout the induction and test sequences, until the end of the test sequence, so that the last gallop of the test sequence was at the extreme left, with the sound at the left ear being $10 \mathrm{~dB}$ more intense. The sound was heard to move gradually from right to left. Unlike the other conditions, in which the three gallops of the test sequence were identical, in this one they continued the right-left movement. We chose this manner of presentation because pretests had shown that, if there was a spatial progression in the induction sequence but not in the test sequence, the auditory system detected the change and reset the accumulation of evidence, favoring stream segregation.

3. Sudden right-to-left. The induction sequence had an apparent location at the extreme right.

4. Sudden center-to-left. The induction sequence was localized in the center of the auditory field.

5. Control. This condition presented a single long continuous burst of dichotically uncorrelated white noise of the same length as the other induction sequences. There is no clear localization of such bursts.

Stimulus parameters. The centered tones were presented at $65 \mathrm{dBA}$ in each ear, and the dichotically uncorrelated white noise was presented at $47 \mathrm{dBA}$ in each ear. The noise level was reduced, relative to that of Experiment 1, because subjects had found the long 51-dBA noise unpleasant in that experiment. Measurement of tone levels was carried out on a continuous tone of $4192 \mathrm{~Hz}$, which had the same frequency as the highest tones of the gallop sequences.

\section{Results}

The dependent variable was the average of the frequency separations used on the last two replications of the trials for each condition. A one-way repeated measures ANOVA was conducted using the GreenhouseGeisser (1959) correction because of inhomogeneity of covariance. A significant effect of induction condition was found $[F(1,19)=38.00, p<.0001]$. The means for

\section{Effect of lateralization change: interaural intensity cues}

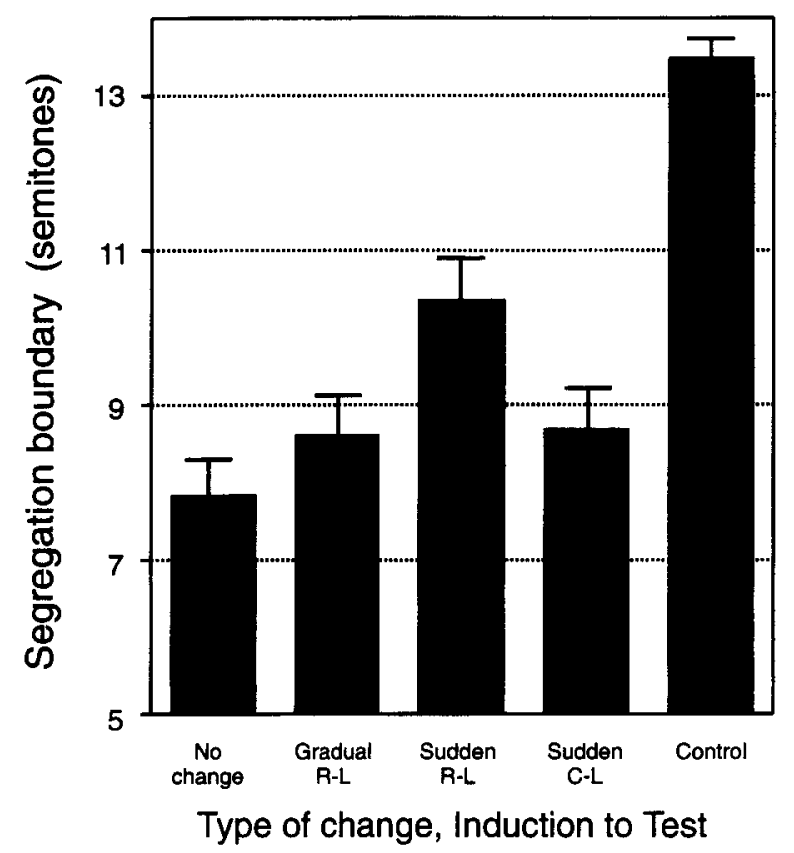

Figure 3. Results of Experiment 2. Segregation boundary following six types of change from induction to test sequence, using interaural intensity cues to control lateralization. Higher values indicate a lower induction of segregation. Vertical bars show standard errors. 
the no change, gradual, sudden right-to-left, sudden center-to-left, and control conditions were (respectively) $7.825,8.6,10.35,8.675$, and 13.475 semitones. The results are shown in Figure 3, with the dependent variable (referred to as the segregation boundary) plotted against the induction condition.

A planned comparison showed that the average of the sudden change conditions was significantly different from the average of the no change and the gradual change conditions $[F(1,19)=23.94, p=.0001]$. However, this result was caused by the difference between the no change and the sudden right-to-left conditions; inspection of the means reveals very similar values for the sudden centerto-left and the gradual change conditions. The second planned comparison showed that the sudden right-to-left and the sudden center-to-left conditions were significantly different in their effects on segregation $[F(1,19)=$ $12.671, p=.0021]$. A Bonferroni adjusted $t$ test was carried out at the .05 level for all pairs of means. The no change, gradual change, and sudden center-to-left conditions were not significantly different from each other, but all induced reliably more segregation than did the sudden right-to-left and the control conditions. The sudden right-to-left condition caused significantly less segregation than did the control condition.

\section{Discussion}

Once more, an effect of sudden change of lateralization on the cumulative effects of streaming was demonstrated. However, because it is impossible to change interaural level differences without also producing at least one within-ear level difference, there is no evidence as to whether the effect is caused by changes in the relations between interaural intensity differences or by changes within each ear taken alone. This is a major limitation of the present experiment. Hafter and Buell $(1985,1990)$ found that the auditory system responds rapidly to changes in binaural information (for both interaural time and intensity differences) after adaptation to unchanging information takes place. Furthermore, their evidence suggested that binaural adaptation is probably caused by monaural peripheral processes that flag the binaural system when transients appear. The response observed in Experiment 2 to sudden changes in binaural information may well be explainable by the same type of monaural processes as those discussed by Hafter and Buell.

Changes in IICs do not affect the resetting of the cumulation of information in quite the same way as do changes in ITCs. In the current experiment, the sudden change from the center to the left of the auditory field did not cause a resetting of segregation. This could mean that the effect of sudden changes in IICs is generally weaker than that of the changes in timing cues, thus requiring a larger change of azimuth to produce the effect. Alternatively, the results may reflect the fact that, in the center-to-left condition, there was a monaural level difference only in the right ear (a drop in level), whereas, for the right-to-left condition, there was a change in both ears (a drop in the right and a rise in the left). The results of Experiment 4 are relevant, and the current experiment will be considered again in the Discussion section of that experiment.

The finding that the change in lateralization from extreme right to extreme left caused a formerly segregated sequence to be heard as coherent again provided evidence that a change in binaural cues (or possibly monaural ones) can effect streaming in the absence of an overall level change. The data added support to the more general experimental hypothesis that sudden changes that indicate large differences in spatial location from one moment to the next are incompatible with continuation of a stream. The larger the change, the less likely are the incoming tones to be grouped with the previously existing stream.

\section{EXPERIMENT 3}

Experiments 1 and 2 gave evidence that sudden changes in either of two individual cues for spatial location-interaural time and intensity differences-can reset the cumulative effects of stream segregation. However, there is a danger in generalizing the findings to sudden changes of spatial location per se, because the experiments were done with single cues over headphones. Stimuli presented over headphones typically appear to come from within the head and lack the usual ancillary information, such as echoes and filter effects of the pinnae. Warren (1982) has advised caution in taking results from spatial location experiments using sounds over headphones and applying these results to sounds heard in the natural environment, because the handling of complex covariation of many cues is an integral part of auditory spatial localization. Middlebrooks and Green (1991) made a similar point, stating: "The restricted experiment provides information about the sensitivity of the listener to a particular cue. It does not provide information about the larger, and more interesting, issue concerning the synthesis of a central image corresponding to the external sound source" (p. 136). For this reason, it was important to test the hypothesis about the effects of changes in spatial location (on the cumulative effects of streaming) by conducting an experiment using stimuli presented over loudspeakers, allowing subjects a full range of auditory cues.

\section{Method}

Although the apparatus was very different, the procedure used in this experiment was similar to those of Experiments 1 and 2. The test sequences, as in those experiments, were presented at the extreme left of the auditory field. There was no concern about possible hemisphere advantages amplifying or weakening the results. because of available data (Robert Zatorre, personal communication, May 1991) showing that the detection of azimuth is very accurate and right-to-left symmetrical in normal subjects listening to sounds from the same speakers and with the same arrangement as those used in Experiment 3. 
Subjects. There were 11 subjects, drawn from a population of students, former students, or staff at McGill University.

Stimuli. The present experiment employed complex tones in order to provide a wide range of frequencies in each tone, so that all spatial cues could be used. The fundamental frequency of the tones varied from 312 to $699 \mathrm{~Hz}$. The first eight harmonics were present, with equal intensities except for the first and eighth, which were attenuated by $6 \mathrm{~dB}$ to prevent them from standing out. Thus, the frequency range for the stimuli in this experiment was from 312 to $5592 \mathrm{~Hz}$. This range provided low frequencies, where interaural timing cues are most effective, and high frequencies, where interaural intensity cues are most effective. A middle range of frequencies, where neither of those cues work well, was also represented, and the highest frequencies were above $4000 \mathrm{~Hz}$, in the range affected by the pinnae.

The gallop patterns had a separation between the fundamental frequencies of the high and low tones of from 5 to 14 semitones, varying in 1-semitone steps. The upper tones of the gallops had a fundamental frequency of $699 \mathrm{~Hz}$. The three-gallop test sequences were, with the exceptions to be described below, always heard at the extreme left of the auditory field. The induction sequence differed from the test sequence only in spatial location.

There were seven different types of induction sequences, chosen to test gradual and sudden changes of spatial location between the induction and the test sequences, as follows.

1. No change. The induction sequence was presented at the extreme left-that is, with the same spatial location as the test sequence. This condition was expected to produce the most segregation of all the induction sequences.

2. Gradual right-to-left. The beginning of the induction sequence was emitted from the speaker at the extreme right and then, on each cycle, the sound was moved by one speaker location (an angle of $15^{\circ}$ ) toward the leftmost speaker, so that the sound was heard to move gradually from the extreme right to the extreme left, reaching the latter position by the end of the test sequence.

3. Gradual center-to-left. The beginning of the induction sequence was emitted from the center speaker, and then the sound moved leftward by one speaker on every second cycle of the sequence. This stimulus was heard to move slowly and smoothly from center to left, even though the sound moved in discrete steps, reaching the extreme left position by the end of the test sequence.

Note that an observation made in pretesting this experiment affected the design of the two gradual change conditions. It seems that the auditory system is sensitive not only to gradualness of change but to uniformity in the rate of change as well. It was originally planned that all the test sequences would contain three 400 msec cycles of the gallop pattern at the extreme left. However, with the gradual change stimuli, this gave rise to a strong perceptible change in the regular pattern of motion. For example, with the gradual right-to-left condition, if there was one 400 -msec gallop cycle at each of Speakers 13-2 and then three cycles at the extreme left (Speaker 1), the listener's attention was drawn to the abrupt cessation in smooth motion. This change in motion caused one of us (W.L.R.) to perceive a resetting of segregation. Whatever mechanism keeps track of stream properties may monitor their rate of change. Accordingly, we employed the stimuli described above for the gradual change induction sequences, to avoid any possible interference from changes in the rate of motion.

4. Sudden right-to-left. The induction sequence came from the speaker at the extreme right.

5. Sudden center-to-left. The induction sequence came from the center speaker.

6. Sudden not-left. This condition contained a sudden change from the induction to the test sequences but was designed to counteract any possible effect from the subjects' learning that the test sequence is always on the extreme left. With complex tones over speakers, the perception of spatial location is so unambiguous that it might have been easy for the subjects to be affected by learning and to have come to hear the spatial shift to the left, especially of the sudden change conditions, as an expected event. This might have allowed them to learn that, in this experimental setting, there existed auditory objects that could shift instantaneously in space to the left. To avoid any possibility of this happening, and, perhaps, interfering with the results, the sixth induction condition was added. In this condition, the induction sequence's location was chosen randomly from the extreme left, the center, or the extreme right. The test sequence's location was also chosen randomly from all the locations other than that of the induction sequence and the extreme left. This was done independently for each presentation and each subject. If subjects remembered the stimuli from one trial to the next, they would, thus, not have had an opportunity to learn that the stimulus always ended up on the left side. The streaming of this sequence was not really of interest, although it was expected to have effects similar to those of the other sudden change conditions.

7. Control. The induction sequence was a single long continuous burst of white noise, of the same length as the other induction sequences, and emitted from the leftmost speaker. It should be pointed out that the uncorrelated dichotic noise in Experiments 1 and 2 was perceived as having a diffuse spatial location, whereas, in the present experiment, the noise was quite localizable. Having uncorrelated white noise coming fiom two speakers (as opposed to headphones) might have led to two separate localizations, one at the left and the other at the right; this was judged to be undesirable.

The amount of segregation induced by the induction sequence was expected to be greatest with the no change condition and least with the control condition. The sudden change conditions were expected to be similar in effect to the control condition, and the gradual change conditions similar to the no change condition.

All tones were presented at $65 \mathrm{dBA}$, and the white noise was presented at $47 \mathrm{dBA}$. Measurement of tone levels was carried out on a continuous complex tone (harmonics $1-8$, as described above) with a fundamental frequency of $699 \mathrm{~Hz}$-that is, a tone the same as the high tones of the gallop sequences. The sampling rate was $16 \mathrm{kHz}$

Training. After listening to the sample sounds, the subjects were given seven practice trials, one of each induction condition type.

Task. As in Experiments 1 and 2, the frequency separation between the high and low tones of the sequences was adjusted during the experiment, but the rules for adjustment were slightly different from those in the previous experiments. On the first trial of each condition, the subject heard sequences with the nine-semitone frequency separation. On subsequent trials of that condition, the frequency separation depended on the response for the previous trial of the same condition. If the response had indicated coherence (subject judged that a gallop was present, giving a rating of 6-8), the frequency separation of the current trial was made one semitone larger. If the response had indicated segregation (subject judged that a gallop was not present, giving a rating of $1-3)$, the frequency separation was made one semitone smaller. However, if the subject had been very unsure (giving a rating of 4 or 5), the frequency separation was not changed. This last rule was added in the present experiment to prevent undue oscillation of the threshold. There were eight replications of each level of the seven induction condition types.

Apparatus. The subjects were tested in the laboratory of Robert J. Zatorre at the Montreal Neurological Institute, whose setup is quite different from that used in Experiments 1,2, and 4, which were carried out in a sound-attenuating test chamber in the McGill Psychology Department. In Zatorre's apparatus, the subjects sit at the center of a semicircular array of 13 small speakers, $1 \mathrm{~m}$ from the subject and $15^{\circ}$ of azimuth apart, all located in a small room (not an acoustic test chamber), as is shown in Figure 4. A diagram of the rating scale is displayed just below the central speaker. The subject's head is supported from behind by a semicircular headrest 


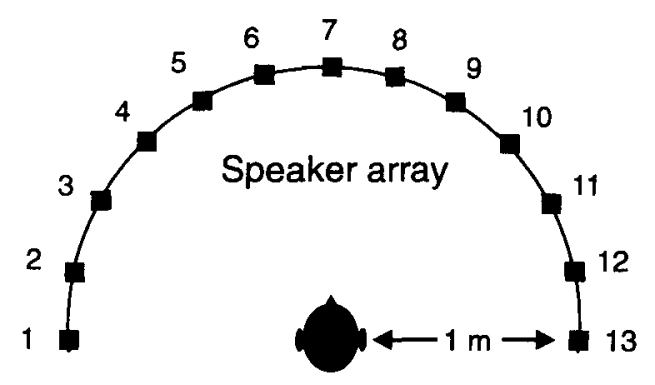

Figure 4. Bird's-eye view of arrangement of speakers and subject in Experiment 3.

so that it faces the center speaker, and the chair is raised or lowered so that the subject's ears are at the level of the middle of the speakers. The walls of the room to the left, right, and rear of the subject are covered with Sonex 2 -in. acoustic panels to attenuate echoes. The computer is located to the left of the speakers, behind a room divider, and is covered with a rigid plastic sound-attenuating enclosure during experiments. Because the apparatus is often used with neurologically impaired subjects, the subjects are not required to use a keyboard to enter their responses. Instead, they call out the ratings, which are then entered by the experimenter, sitting several feet beyond speaker number 4 , who enters the responses on a computer keyboard. This apparatus has been used in many experiments with brain-damaged and normal subjects. The latter are generally quite accurate in localizing the sounds in space (Zatorre, Ptito, \& Villemure, 1995).

The signals were low-pass filtered at $8000 \mathrm{~Hz}$, using a TTE filter (passive Tchebychev) having 60-dB attenuation at $11.2 \mathrm{kHz}$, THD $<0.1 \%$. They were amplified by a Harmon/Kardon HK6100 amplifier and presented over D-BOX Model T-55AS speakers.

\section{Results}

The dependent variable was the average of the frequency separations used on the last two replications of the trials for each condition. A one-way repeated measures ANOVA was conducted using the Greenhouse-Geisser (1959) correction because of inhomogeneity of covariance. A significant effect of induction sequence type was found $[F(1,10)=21.372, p<.01]$. The means for the no change, gradual right-left, gradual center-left, sudden right-left, sudden center-left, sudden not-left, and control induction sequences were (respectively) 7.91, 9.59, 9.46, 13.41, 13.27, 12.18, and 13.36. The results are shown in Figure 5.

A planned comparison showed that the average of the sudden change conditions was significantly different from the averages of the no change and the gradual change conditions $[F(1,10)=33.5, p=.0002]$. A second planned comparison showed that the sudden right-left and the sudden center-left conditions were not significantly different in their effects on segregation $[F(1,10)=0.214$, $p>.5]$. A Bonferroni adjusted $t$ test was carried out at the .05 level for all pairs of means. The no change and the two gradual change conditions were not reliably different from one another, but all had a significantly smaller segregation boundary than did the control, the sudden rightleft, or the sudden center-left conditions.
The means for the control, sudden right-left, sudden center-left, and sudden not-left conditions were all close to the maximum value of 14 , suggesting that there may have been ceiling effects in the experiment. Perhaps, if ceiling effects had been avoided, we might have found significant differences among these conditions. However, this would simply have shown that, even with sudden changes, some induction of streaming could occur. It would leave unaffected the conclusion that sudden changes induce less streaming than do no change or gradual changes.

\section{Discussion}

This experiment, with stimuli heard over loudspeakers, showed a strong effect of a sudden change of spatial location on streaming. There was a clear separation of the means for sudden and gradual shifts of location. This is good evidence that the results of Experiments 1 and 2 were not simply artifacts of stimuli presented over headphones. Not all changes have the same effects. In the case of the gradual change stimuli, it appears that each new sound was incorporated into the stream of gallops and its location treated as an update of the location of that stream. Although the spatial location moved in discrete steps $\left(15^{\circ}\right.$ or $\left.30^{\circ}\right)$, these seemed to be small enough and regular enough that they allowed the gallops to be incorporated into a single stream that had a smooth mo-

\section{Effect of spatial change: Loudspeaker array}

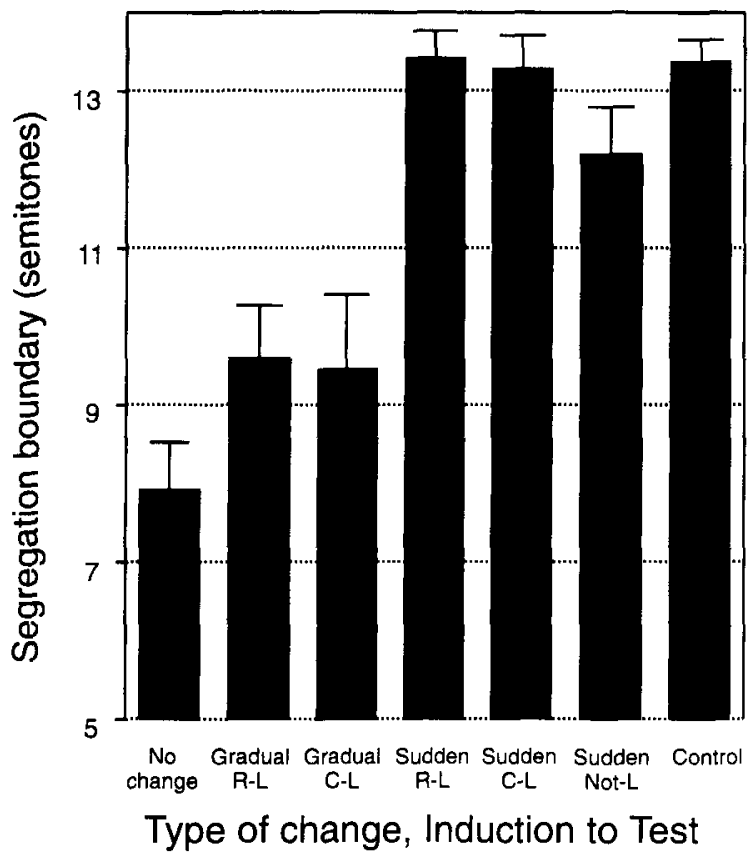

Figure 5. Results of Experiment 3. Segregation boundary following seven types of change from induction to test sequence, using sounds presented over loudspeakers. Higher values indicate a lower induction of segregation. Vertical bars show standard errors. 
tion. Therefore, they did not cause a reset of the accumulation of stream information.

Figure 5 shows a value for the gradual change conditions slightly different from that of the no change condition, even though the effect was not statistically significant with a sample size of 11 . Significant or not, the gradual changes did not cause much loss of segregation.

\section{EXPERIMENT 4}

Experiments 1-3 demonstrated that sudden changes of spatial location can have an effect on streaming. It was also possible that sudden changes of overall sound level could have similar effects. In Experiments 2 and 3, when there was a sudden shift in location, there was, in at least one ear, a sudden change in intensity between induction and test sequences. This may have played a role in the observed resetting. Experiment 4 was designed to test systematically the effects of intensity change.

\section{Method}

The procedure was very similar to that of the last three experiments. Sudden and gradual changes of both increases and decreases in intensity were tested.

Subjects. There were 20 adult subjects, drawn from a university population.

Stimuli. All tones were pure sinusoids, in the range 589$1248 \mathrm{~Hz}$. The frequencies were chosen in a range in which equal loudness curves are fairly flat. The sampling rate was $20 \mathrm{kHz}$. The test sequences' gallop patterns had a separation between high and low tones of $5,6,7,8,9,10,11,12$, or 13 semitones. The high tones of the gallop sequences in both the induction and the test sequences were always at $1248 \mathrm{~Hz}$. All stimuli were diotic, including the white noise control condition.

The induction sequence preceding each test sequence contained cycles of the gallop pattern, with a frequency separation between the high and low tones matching that of the test sequence, and differing from the test sequence only in intensity. There were six different types of induction sequences, chosen to test gradual and sudden changes of sound level between the induction sequence and the test sequence.

1. No change. The induction sequence was presented at the same intensity as the test sequence, $65 \mathrm{dBA}$. This condition was expected to produce the most segregation of all the induction sequences.

2. Gradual louder. The beginning of the induction sequence was at $59 \mathrm{dBA}$. The sound level was constant within each cycle, changing only between cycles (for a total of 14 changes), so that the sound was heard to become gradually louder. This gradual increase started at the first cycle of the induction sequence and continued changing smoothly until the final cycle of the test sequence, at which it reached $71 \mathrm{dBA}$.

3. Gradual softer. This was a reverse of the second condition: The beginning of the induction sequence was at $71 \mathrm{dBA}$, and the sound level was lowered throughout the induction and test sequences, reaching $59 \mathrm{dBA}$ at the end of the test sequence.

4. Sudden louder. The induction sequence was at $59 \mathrm{dBA}$, and the test sequence was at $71 \mathrm{dBA}$.

5 . Sudden softer. The induction sequence was at $71 \mathrm{dBA}$, and the test sequence was at $59 \mathrm{dBA}$.

6. Control. The induction sequence was a single long continuous burst of white noise (at $47 \mathrm{dBA}$ ), the same length as the other induction sequences, and the test sequence was a gallop presented at $65 \mathrm{dBA}$

\section{Effect of intensity change: binaural signal}

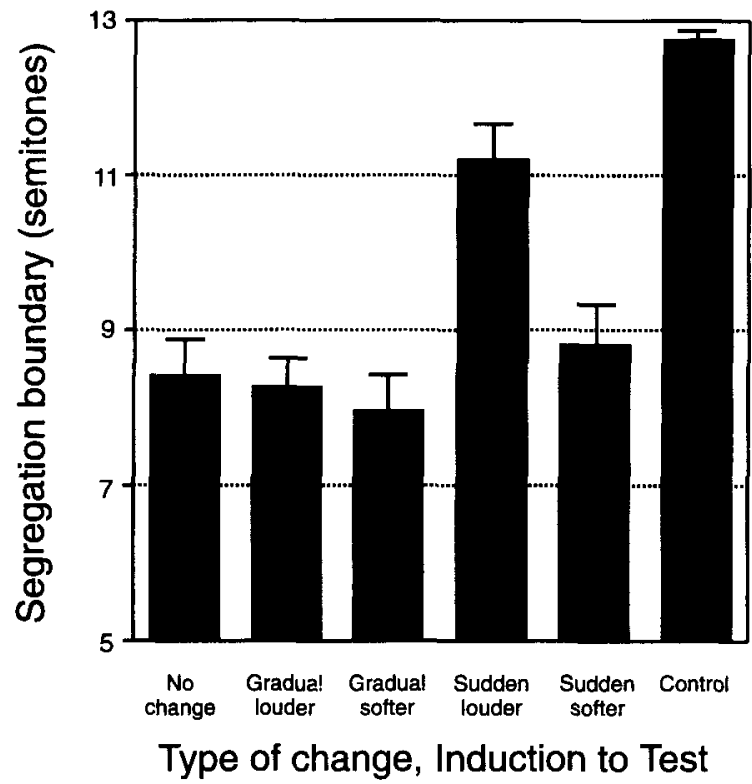

Figure 6. Results of Experiment 4. Segregation boundary following six types of intensity change from induction to test sequence. Higher values indicate a lower induction of segregation. Vertical bars show standard errors.

The amount of segregation induced by the induction sequence was expected to be greatest with the no change condition and least with the control condition. Both of the sudden change conditions were expected to be similar in effect to the control condition, and the gradual change conditions similar to the no change condition. Measurement of tone levels was carried out on a continuous tone of $1248 \mathrm{~Hz}$.

Procedure and Apparatus. There were nine replications of each level of the six induction condition types. Synthesis, filtering, and playback (over headphones) were the same as those in Experiment 1.

\section{Results}

For each condition, the average of the frequency separations used on the last two replications of the trials for that condition was the dependent variable. A one-way (induction condition type) repeated measures ANOVA was conducted, using the Greenhouse-Geisser (1959) correction because of nonhomogeneity of covariance. A significant effect of induction condition type was found $[F(1,19)=31.658, p<.01]$. The means for the no change, gradual louder, gradual softer, sudden louder, sudden softer, and control conditions were (respectively) 8.40 , $8.25,7.95,11.20,8.80$, and 12.75 semitones. The results are shown in Figure 6.

A planned comparison showed that the average segregation boundary over the sudden change conditions was significantly larger than the average of the no change and the gradual change conditions $[F(1,19)=25.38, p<$ $.0001]$. This result, however, was caused mainly by the difference between the no change and the sudden louder 
conditions; inspection of the means revealed very similar values for the sudden softer and the gradual change conditions. The second planned comparison confirmed the inspection of the means: Contrary to our hypothesis, the sudden louder and the sudden softer conditions were significantly different in their effects on segregation $[F(1,19)=13.20, p=.0018]$. A Bonferroni adjusted $t$ test was performed at the .05 level for all pairs of means. The no change, gradual change (louder and softer), and sudden softer conditions were not significantly different in effect from each other, but all gave reliably lower boundaries than did the sudden louder condition. The sudden louder condition, in turn, caused a significantly lower boundary than did the control condition.

\section{Discussion}

Changes in loudness are different from changes in spatial location in their effects on stream segregation. One illustration is the contrast between the sudden change conditions of Experiments 3 and 4. The former showed that any changes of spatial location that were perceived as discontinuous would result in coherence of the test sequence. In Experiment 4, a comparison of the sudden softer and sudden louder conditions shows that, for intensity, equally large sudden changes in different directions do not have the same effects. A sudden increase seems to restore the one-stream interpretation, allowing the gallop percept to reemerge, but other loudness changes do not interfere with the cumulative effects of streaming. The need for a large increase in the intensity of stimulation in order to regain the gallop percept is consistent with the adaptation of some as yet unknown process. Alternatively, a mechanism that checked for discontinuities in stream properties might respond primarily to sudden increases in intensity. This would make sense in evolutionary terms. Although sound sources in the environment often change suddenly in intensity, a decrease could not signal the presence of an additional (new) sound source, whereas a sudden increase could very well do so. On the other hand, a decrease might signal the end of one louder sequence that had been masking a weaker one, so that the second's properties now began to dominate the spectrum.

The effects of sudden drops of intensity may be real but too weak to be observed in this experiment. One relevant observation in this experiment was not reported in the Results section: There were great individual differences in the pattern of responses. When individual results were plotted, and compared visually to the overall results shown in Figure 6, four subjects showed a pattern different from the overall pattern. Their segregation boundaries for the sudden softer condition were similar to those for the sudden louder condition. This could indicate that there are differences in auditory scene analysis processes among individuals. Another possibility is that sufficiently large decreases in sound level could have the same effect as moderate increases; perhaps the 4 subjects who showed an effect of decreases in level reflect quantitative, rather than qualitative, differences in responding, as compared with the other subjects. The results of the diotic gallop condition of Experiment 3 of Rogers and Bregman (1993) are pertinent, because there the transition from the induction to the test sequence involved a very large drop in sound level at the right ear, with no level change at the left ear, yet resetting was observed.

It is worthwhile to reexamine some of the conditions from Experiment 2, where changes of lateralization by IICs were tested. In that experiment, the sudden centerleft condition was not significantly different from the no change condition, whereas the sudden right-left condition was equivalent to the control condition. Recall that only in the sudden right-left condition was there an increase in monaural level. Therefore, since in Experiment 4, only increases in level could be shown to cause resetting of segregation, it appears that the results of Experiment 2 might be explainable purely by monaural level differences.

A criticism that could be made of this experiment is that the test sequence was not identical in all the conditions. Perhaps there should have been two no change conditions and two control conditions, one each with sound levels of the gallop pattern set to 71 and $59 \mathrm{dBA}$. The choice of an intermediate loudness for the no change and the control conditions in this experiment was perhaps what explains the sudden-louder condition's yielding a slightly lower boundary than did the control condition. However, the most important aspect of the experiment was the test of the hypothesis regarding the differences between sudden and gradual changes of sound level. Here the gradual change and sudden change conditions acted as controls for each other.

To answer any queries about the validity of the controls, an auxiliary experiment was conducted, with only 2 subjects, in order to determine whether the pattern of results would be the same when all the test sequences had the same sound level. The test sequences were presented at $65 \mathrm{dBA}$, the gradual louder and sudden louder sequences were begun at $59 \mathrm{dBA}$, and the gradual softer and sudden softer sequences were begun at $71 \mathrm{dBA}$. All other details were identical to those of Experiment 4.

Despite the smaller changes of sound level in the experimental conditions ( $6 \mathrm{~dB}$ instead of 12$)$, the pattern of results for each subject was the same as the overall pattern of Experiment 4. The means for the no change, gradual louder, gradual softer, sudden louder, sudden softer, and control conditions were (respectively) 7.5, 7.5, $7.5,11.5,7.5$, and 13. Therefore, one can feel confident that the original choice of stimuli did not discredit the experiment. The sudden louder and control conditions were as different for the 2 subjects of the auxiliary experiment as for the subjects in the main experiment. This implies that sudden increases in loudness are not as effective as sudden changes in spatial location in their consequences on segregation. 


\section{GENERAL DISCUSSION}

To summarize our results, Experiments 1-3 showed similar effects of the type of change of perceived location between induction and test sequences, regardless of whether this was signaled over headphones by ITCs or IICs or signaled in a free field in which different loudspeakers presented the signal. When there was no spatial change, the induction of segregation in the test sequence was greatest; gradual changes induced less segregation; and sudden changes induced the least. These effects were most pronounced when all the cues for location were present (using the loudspeaker array). Experiment 4 showed that a sudden rise in intensity between induction and test sequences also tended to dissociate the two sequences, so that there was little induction of segregation. This suggests that, in a normal environment, when a sound from one location follows a sound from another, the rise in intensity at one ear may be playing a role in the resetting of streaming. However, the effectiveness of sudden changes in the interaural timing cues of Experiment 1, which were constant in intensity, shows that the effect of spatial change is not entirely due to this rise in intensity at one ear.

A suddenness-of-change effect on the formation of auditory streams was demonstrated earlier by Bregman and Dannenbring (1973). They showed that high and low tones were less likely to segregate if they were connected by gliding frequency transitions. However, their demonstration was different in an important way from ours. In their demonstration, the gradualness of change was along the frequency dimension, and it was on that same dimension that stream segregation occurred (high tones segregated from low ones). We can summarize this by saying that the gradualness of change was on the streamdefining dimension. In the present experiment as well, segregation took place on the frequency dimension, but the suddenness of change that we examined was on a non-stream-defining dimension-spatial location.

Why should the suddenness of such an irrelevant change make a difference? To set the stage for our explanation, we must suppose that, centered on each value along some property dimension (e.g., at particular values of frequency on the frequency dimension), there are mechanisms that accumulate information about the occurrence of recent events having that value. From a functional point of view, we can say that evidence is accumulated about a set of environmental events that have a certain property. This evidence could conceivably be held in the form of increases or decreases in the activation of a neural circuit or in the status of some neurochemical process, but, since our preference is to offer explanations that are purely functional in nature, we make no claims about the underlying process. We think of it simply as a quantity that can rise and fall. The assumption of a buildup and decay of evidence--seen as level of activation-is built into the neural processing model of Beauvois (1993).
Each new stimulus of the right value or range of values (e.g., a tone that falls within a narrow range of frequencies) adds to that quantity until some maximum is reached, a process that takes at least $4 \mathrm{sec}$, and the absence of events in that range of values causes the quantity to drop to zero, a process that also takes at least $4 \mathrm{sec}$ (Bregman, 1978).

This explains why the high tones in our experiment were segregated from the low ones. But it does not explain why an apparently irrelevant dimension such as spatial location or intensity should make a difference in the segregation of high and low tones. The explanation may be that, although from the experimenter's perspective location is irrelevant, the auditory system has no way of knowing this in advance. The values for which evidence is being accumulated may not just be regions on the frequency dimension but regions in a multidimensional space that includes the dimensions of spatial position and intensity among others - that is, evidence is accumulated that certain values of frequency, location, intensity, and so forth, have recently occurred in combination.

With this in mind, two possible explanations for our results occur to us. The first is as follows: If the location of the induction sequence gradually approaches that of the test sequence, as in the gradual change conditions, this may allow the evidence accumulation processes for high and low tones centered at the position of the test sequence to start to respond to some of the later gallops in the induction sequence before the test sequence begins. This means that the segregation tendency starts to build up earlier at the new location in the gradual change conditions than in the sudden change ones. This explanation sees the effect of gradual change as doing nothing other than bringing the later gallops of the induction sequence closer to the location of the test sequence; suddenness itself is not important.

The alternative explanation sees suddenness and gradualness as having effects in their own right. When a newly arriving stimulus represents a sudden change in the properties of the incoming sound, this suddenness may act directly to define an acoustic boundary (much in the way that visual boundaries were said to be established by the Gestalt psychologists). This establishing of a boundary might set in motion a new evidence accumulation process at the combination of values that are present in the new stimulus. On the other hand, when changes are gradual, the accumulated evidence for a stream may be transferred to the location of the next stimulus with little loss in strength, as evidenced by the strong induction observed in the gradual change conditions of the present experiments.

If some types of sudden change cause the auditory streaming mechanism to recompute stream properties and to reevaluate grouping, this would be a useful strategy: When no discontinuities are detected, it is likely that the signal is still coming from the same sound source, so the auditory system can slow down its computation of the properties of sound. Hafter and Buell (1990) have 
suggested that the auditory computation of spatial location relies on "a sample-on-demand process that extracts the interaural cues only when there is a new signal" (p. 811), thus reducing the load on central processing. They were concerned only with the updating of binaural information for purposes of the localization itself, but the evidence presented in Experiments $1-3$ suggests the existence of a sample-on-demand system in auditory scene analysis in general.

To summarize, there are two alternative explanations for the effects of gradual change in our experiment: (1) The role of gradual change is to simply bring the later gallops of the induction sequence nearer to the region sampled by the evidence accumulation process centered on the location of the test sequence, or (2) the gradual change prevents a discontinuity that signals the beginning of a new sound -producing event in the environment. It is not clear to us how these two alternatives could be distinguished by behavioral studies. Perhaps the observation of a mismatch negativity (MMN) response in event related potentials gathered by electroencephalography might be helpful in distinguishing them. If an MMN response were observed when perceived location changed suddenly, it would tend to support the second explanation.

The effects of sudden changes in resetting perceptual analyses have been observed in other contexts than that in the Hafter and Buell (1990) study mentioned earlier. Darwin and Bethell-Fox (1977) showed that, in synthesized speech, a sudden change in fundamental frequency created a boundary across which phonetic interpretation processes would not integrate spectral information. Bregman, Ahad, Kim, and Melnerich (1994) and Bregman, Ahad, and Kim (1994) showed that the suddenness of changes in amplitude affected the tendency of a particular frequency to stand out from a background of other pitches. Perhaps the effects of suddenness of change observed in the present studies fall into the same family. It may be that sudden changes, in general, tell the auditory system that new events are occurring and that it should start up a new analysis rather than update an old one.

\section{REFERENCES}

ANSTIS, S., \& SAIDA, S. (1985). Adaptation to auditory streaming of frequency-modulated tones. Journal of Experimental Psychology: Human Perception \& Performance, 11, 257-271.

Atrneave, F., \& Olson, R. K. (1971). Pitch as a medium: A new approach to psychophysical scaling. American Journal of Psychology, 84, 147-166.
Beauvois, M. W. (1993). Computer simulation of auditory stream segregation in sequential-tone sequences. Journal of the Acoustical Society of America, 93, 2308.

Bregman, A. S. (1978). Auditory streaming is cumulative. Journal of Experimental Psychology: Human Perception \& Performance, 4. 380387.

Bregman, A. S., AHAD, P., \& Kim, J, (1994). Resetting the pitch analysis system: 2 . Role of sudden onsets and offsets in the perception of individual components in a cluster of overlapping tones. Journal of the Acoustical Society of America, 96, 2694-2703.

Bregman, A. S., Ahad, P., Kim, J., \& Melnerich, L. (1994). Resetting the pitch-analysis system: I. Effects of rise times of tones in noise backgrounds or of harmonics in a complex tone. Perception \& Psychophysics, 56, 155-162.

Bregman, A. S., \& Dannenbring, G. L. (1973). The effect of continuity on auditory stream segregation. Perception \& Psychophysics, 13, 308-312.

Darwin, C. J., \& Bethell-Fox, C. E. (1977). Pitch continuity and speech source attribution. Journal of Experimental Psychology: $\mathrm{Hu}$ man Perception \& Performance, 3, 665-672.

Greenhouse, S. W., \& Geisser, S. (1959). On methods in the analysis of profile data. Psychometrika, 24, 95-112.

HAFTER, E. R., \& BuEll, T. N, (1985). The importance of transients for maintaining the separation of signals in auditory space. In M. I. Posner and O. S. M. Marin (Eds.), Attention and Performance X/ (pp. 337354). Hillsdale, NJ: Erlbaum.

HAFTer, E. R., \& Buell, T. N. (1990). Restarting the adapted binaural system. Journal of the Acoustical Society of America, 88, 806-812.

HENKE, W. L. (1987). MITSYN: A Coherent Family of Command-Level Utilities for Time Signal Processing (Version 6.1) [Computer software]. Belmont, Mass: Author.

JONES, M. R. (1976). Time, our lost dimension: Toward a new theory of perception, attention, and memory. Psychological Review, 83.323355.

Middebrooks, J. C., \& Green, D. M. (1991). Sound localization by human listeners. Annual Review of Psychology, 42, 135-159.

MOORE, B. C. 3. (1982). An introduction to the psychology of heuring. New York: Academic Press.

Rogers, W. L., \& Bregman, A. S. (1993). An experimental evaluation of three theories of auditory stream segregation. Perception \& Psychophysics, 53, 179-189

VAN NoORden, L. P. A. S. (1975). Temporal coherence in the perception of tone sequences. Unpublished doctoral dissertation, Eindhoven University of Technology.

VAN NOORDEN, L. P. A. S. (1977). Minimum differences of level and frequency for perceptual fission of tone sequences $A B A B$. Journal of the Acoustical Society of America, 61, 1041-1045.

Warren, R. M. (1982). Auditory perception: A new synthesis. New York: Pergamon.

Zatorre, R. J., Ptito, A., \& Vil.lemure, J.-G. (1995). Preserved auditory spatial localization following cerebral hemispherectomy. Brain, 118. 879-889.

(Manuscript received November 18, 1996; revision accepted for publication October 30, 1997.) 